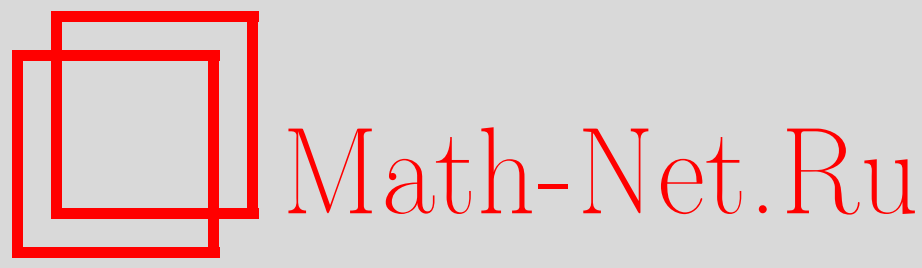

Б. А. Зон, А. С. Корнев, Квазиклассическое приближение для уравнения Дирака в центральном поле, $T M \Phi$, 2012, том 171, номер 1, 65-78

DOI: https://doi.org/10.4213/tmf6919

Использование Общероссийского математического портала Math-Net.Ru подразумевает, что вы прочитали и согласны с пользовательским соглашением http://www.mathnet.ru/rus/agreement

Параметры загрузки:

IP : 54.166 .219 .16

26 апреля 2023 г., 16:55:57

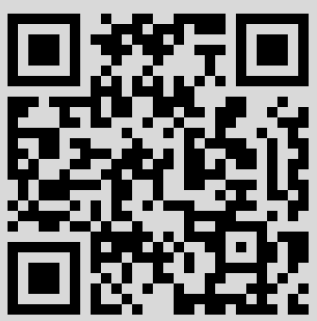




\title{
КВАЗИКЛАССИЧЕСКОЕ ПРИБЛИЖЕНИЕ УРАВНЕНИЯ ДИРАКА В ЦЕНТРАЛЬНОМ ПОЛЕ
}

\begin{abstract}
Развито квазиклассическое приближение уравнения Дирака в центральном поле с кулоновской асимптотикой. Получены релятивистские квазиклассические фазы рассеяния и уровни энергии водородоподобных ионов, а также квазиклассическое выражение для мультипликативной константы асимптотики волновой функции валентного электрона в релятивистском многозарядном ионе, имеющей важное значение в теории квантового дефекта.
\end{abstract}

Ключевые слова: уравнение Дирака, квазиклассическое приближение, метод квантового дефекта.

\section{1. ВВЕДЕНИЕ}

Несмотря на значительные успехи, достигнутые при численном решении волновых уравнений, приближенные методы квантовой механики продолжают оставаться полезными, а часто и единственно возможными методами теоретического анализа. Это относится и к квазиклассическому приближению [1], в рамках которого, в частности, описывается туннельный эффект, ответственный за образование многозарядных ионов в сильном лазерном поле (см. теоретические работы [2], [3], в которых имеются ссылки на экспериментальные результаты).

Для ионов большой кратности в вероятности туннельного эффекта начинают проявляться релятивистские эффекты. Их рассмотрению для водородоподобных ионов посвящены работы [4], [5]. Обобщение этих результатов на более сложные ионы требует релятивистского обобщения известного в атомной и молекулярной физике метода квантового дефекта (см. книгу [6], а также уточнение теории, данное в статье [7]). Частично такое обобщение было получено в работе [8]. Для применения этих результатов к туннельному эффекту необходимо, кроме того, релятивистское описание связанного состояния электрона в многозарядном ионе. Иными словами, требуется знание волновой функции электрона в многозарядном ионе в рамках метода квантового дефекта в квазиклассическом приближении.

Квазиклассическому приближению уравнения Дирака посвящена работа [9]. Однако предложенный в этой работе метод не позволяет добиться совпадения фаз

* Воронежский государственный университет, Воронеж, Россия. E-mail: a-kornev@yandex.ru 
рассеяния медленного электрона на ионе с точными фазами рассеяния, которые в теории квантового дефекта играют определяющую роль [6]. Это, в свою очередь, связано с тем, что в работе [9] не рассматривался важный вопрос о релятивистском обобщении хорошо известной в нерелятивистской квантовой механике квазиклассической замены

$$
l(l+1) \rightarrow \mathcal{L}_{l}^{2}=\left\{\begin{array}{lll}
(l+1 / 2)^{2}, & \text { если } & l \neq 0, \\
0, & \text { если } & l=0
\end{array}\right.
$$

где $l$ - орбитальный момент электрона. Релятивистское соотношение, аналогичное (1), можно получить именно из условия совпадения точной фазы рассеяния с ее квазиклассическим выражением, как и в нерелятивистском случае [1]. При этом спектр связанных состояний получается из обычного условия квантования Бора-Зоммерфельда.

При проведении вычислений, необходимых для обобщения соотношения (1) на релятивистский случай, достаточно ограничиться рассмотрением волновых функций только в асимптотически далекой области. Но именно эта асимптотическая область, где потенциал атомного или молекулярного остова можно считать кулоновским, является ответственной за формирование электромагнитных характеристик атома или молекулы в методе квантового дефекта, в том числе вероятности туннельного эффекта во внешнем электрическом поле. Фактическое совпадение областей применимости квазиклассического приближения и метода квантового дефекта было впервые отмечено в работе [10].

Релятивистскому обобщению замены (1) посвящен раздел 2 настоящей работы. В разделе 3 показано, что с учетом такой подстановки обычные условия квантования Бора-Зоммерфельда приводят к точным выражениям для энергий связанных состояний релятивистского водородоподобного атома. Полученные результаты применяются далее в разделе 4 к нахождению постоянной, определяющей асимптотическое поведение волновой функции связанного электрона на больших расстояниях от ядра. Эта постоянная наряду с энергией связи электрона и квантовыми числами, характеризующими движение в пространстве угловых переменных, определяет вероятность туннелирования в сильном лазерном поле.

В настоящей работе всюду, где не оговорено особо, используется система единиц, в которой $c=\hbar=m=1(m-$ масса электрона $), \alpha=e^{2} / \hbar c \simeq 1 / 137$.

\section{2. РЕЛЯТИВИСТСКОЕ ОБОБЩЕНИЕ ЗАМЕНЫ (1)}

В нерелятивистской теории квантового дефекта энергия связанного электрона $E_{n l}^{(\text {nonrel) }}$ в сложном атоме определяет эффективное главное квантовое число $\nu_{n l}$ по той же формуле, что и для водородоподобного атома:

$$
E_{n l}^{(\text {nonrel })}=-\frac{1}{2}\left(\frac{\alpha Z}{\nu_{n l}}\right)^{2}
$$

где $Z$ - заряд остаточного иона, $n=1,2, \ldots$ - главное квантовое число, а $l=$ $0, \ldots, n-1$ - орбитальное квантовое число. Квантовый дефект задается формулой $\mu_{n l} \stackrel{\text { def }}{=} n-\nu_{n l}$. В нерелятивистском водородоподобном ионе $\nu_{n l}=n, \mu_{n l}=0$. 
Естественно определить эффективное главное квантовое число релятивистского электрона $\nu_{n \varkappa}$ через его полную энергию в связанном состоянии в потенциале с кулоновской асимптотикой $E_{n \varkappa}$ следующим соотношением:

$$
E_{n \varkappa}=\left[1+\left(\frac{\alpha Z}{\nu_{n \varkappa}}\right)^{2}\right]^{1 / 2}
$$

где

$$
\varkappa=\left\{\begin{array}{lll}
-l-1, & \text { если } & j=l+1 / 2, \\
l, & \text { если } & j=l-1 / 2,
\end{array}\right.
$$

$j$ - квантовое число, определяющее полный момент электрона, равный векторной сумме орбитального и спинового моментов. Нетрудно видеть, что $\varkappa= \pm 1, \pm 2, \ldots$.

Нерелятивистская энергия (2) получается из формулы (3) после вычитания энергии покоя электрона и замены индекса $\varkappa \rightarrow l$ в виде первого неисчезающего члена разложения по параметру $\alpha Z \ll 1$.

Учитывая точную формулу для спектра релятивистского атома водорода [11], определим релятивистский квантовый дефект:

$$
\mu_{n \varkappa} \stackrel{\text { def }}{=} n-\nu_{n \varkappa}+\gamma-|\varkappa|, \quad \gamma=\sqrt{\varkappa^{2}-(\alpha Z)^{2}} .
$$

В релятивистском водородоподобном ионе $\nu_{n \varkappa}=n+\gamma-|\varkappa|$ и $\mu_{n \varkappa}=0$.

Волновая функция релятивистского электрона, связанного в ионе, удовлетворяет уравнению Дирака и является биспинором:

$$
\Psi_{q j l m}(\mathbf{r})=\frac{1}{r}\left(\begin{array}{c}
f_{q \varkappa}(r) \Omega_{j l m}(\mathbf{r} / r) \\
i g_{q \varkappa}(r) \Omega_{j l^{\prime} m}(\mathbf{r} / r)
\end{array}\right), \quad l^{\prime}=2 j-l,
$$

где

$$
\Omega_{j l m}(\mathbf{n})=\sum_{m_{l}, m_{s}} C_{l m_{l} m_{s} / 2}^{j m} Y_{l m_{l}}(\mathbf{n}) \chi_{m_{s}},
$$

$C$ - коэффициенты Клебша-Гордона, $\chi_{ \pm 1 / 2}$ - спиноры Паули. Радиальные зависимости большой и малой компонент волновой функции (4) даются соответственно функциями $f_{q \varkappa}(r)$ и $g_{q \varkappa}(r)$. Их асимптотический вид приведен, например, в работе [8]:

$$
\lim _{r \rightarrow \infty}\left\{\begin{array}{l}
f_{q \varkappa}(r) \\
g_{q \varkappa}(r)
\end{array}\right\}=\sqrt{\frac{1 \pm E_{n \varkappa}}{2}} C_{q \varkappa} \sqrt{q}(q r)^{\nu_{n \varkappa}} e^{-q r},
$$

где волновое число $q$ связано с энергией $E_{n \varkappa}$ соотношением $q^{2}=1-E_{n \varkappa}^{2}$.

Построение релятивистского квазиклассического приближения удобно проводить на основе квадрированного уравнения Дирака [1], [11]. Радиальная часть этого уравнения за пределами ионного остова имеет вид

$$
\frac{d^{2}}{d r^{2}} R_{E \varkappa}(r)+\left[-\frac{\omega(\omega-1)}{r^{2}}+\frac{2 \alpha Z E}{r}+E^{2}-1\right] R_{E \varkappa}(r)=0,
$$

где $\omega=\gamma-|\varkappa|+l+1$. Поскольку из формулы (5) следует, что вдали от ядра обе компоненты $f_{q \varkappa}(r)$ и $g_{q \varkappa}(r)$ отличаются друг от друга лишь постоянным множителем, в уравнении (6) обе эти компоненты обозначены как $R_{E \varkappa}(r)$. 
В связанных состояниях, при $E=E_{n \varkappa}$, уравнение (6) приводится к виду, формально совпадающему с нерелятивистским уравнением Шредингера:

$$
\frac{d^{2}}{d r^{2}} R_{q \varkappa}(r)+\left[-\frac{\omega(\omega-1)}{r^{2}}+\frac{2 \alpha \mathcal{Z}}{r}-\frac{\alpha^{2} \mathcal{Z}^{2}}{\nu_{n \varkappa}^{2}}\right] R_{q \varkappa}(r)=0 .
$$

Это уравнение отличается от радиального уравнения Шредингера заменами

$$
Z \rightarrow \mathcal{Z}=Z\left[1+\left(\frac{\alpha Z}{\nu_{n \varkappa}}\right)^{2}\right]^{-1 / 2}, \quad l \rightarrow \omega-1,
$$

где индексы $(n, \varkappa)$ у величины $\mathcal{Z}$ для краткости опущены. Таким образом, уравнение (7) описывает движение с нецелым моментом

$$
\lambda=\omega-1=\gamma-|\varkappa|+l,
$$

и для него можно использовать обычный нерелятивистский формализм квазиклассического приближения.

Классический импульс, соответствующий уравнению (7), имеет вид

$$
p(r)=\left[-\frac{\Lambda_{\varkappa}^{2}}{r^{2}}+\frac{2 \alpha \mathcal{Z}}{r}-\frac{\alpha^{2} \mathcal{Z}^{2}}{\nu_{n \varkappa}^{2}}\right]^{1 / 2} .
$$

Как будет показано ниже, константа $\Lambda_{\varkappa}$, являющаяся релятивистским аналогом константы $\mathcal{L}_{l}$ из формулы $(1)$, не зависит от энергии электрона и параметров оболочки остаточного иона. Ее значение находится из условия совпадения квазиклассической фазы рассеяния с точным значением фазы [12]. С этой целью исследуем асимптотическое решение уравнения Дирака в непрерывном спектре с энергией $E^{2}=1+k^{2}$. В этом случае радиальное решение уравнения Дирака будет содержать обе компоненты $f_{k \varkappa}(r)$ и $g_{k \varkappa}(r)$, которые, в отличие от связанных состояний, не сводятся друг к другу в асимптотически далекой области с помощью умножения на постоянный множитель. Их можно получить аналитическим продолжением соответствующих компонент $f_{q \varkappa}(r)$ и $g_{q \varkappa}(r)$ в дискретном спектре в соответствии с правилом [13]

$$
q=\frac{\alpha \mathcal{Z}}{\nu_{n \varkappa}} \rightarrow i k
$$

Следует заметить, что из решения (5) исключены нефизические экспоненциально растущие на бесконечности компоненты, пропорциональные $e^{q r}$. При аналитическом продолжении (10) эти растущие компоненты необходимо учитывать. Приведем асимптотический вид (при $k r \rightarrow \infty$ ) решения уравнения Дирака в непрерывном спектре [13]:

$$
f_{k \varkappa}(r) \sim \sqrt{\frac{E+1}{2}} \sin \left[\Phi_{k \varkappa}(r)\right], \quad g_{k \varkappa}(r) \sim \sqrt{\frac{E-1}{2}} \cos \left[\Phi_{k \varkappa}(r)\right],
$$

где

$$
\Phi_{k \varkappa}(r)=k r+\eta \ln (2 k r)-\arg \Gamma(\gamma+1+i \eta)+\frac{1}{2} \arg \frac{\varkappa-i \eta / E}{\gamma-i \eta}-\frac{\pi}{2} \gamma
$$

и $\eta=\alpha Z E / k$. 
При наличии короткодействующего потенциала ионного остова, убывающего на бесконечности быстрее чем $r^{-2}$, к фазе $(11)$ добавляется сдвиг $\delta_{k \varkappa}$, который при низких энергиях (при $q \rightarrow 0, n \rightarrow \infty$ ) связан с квантовым дефектом $\mu_{n \varkappa}$ релятивистской формулой Ситона [8]. Она полностью аналогична соответствующей нерелятивистской формуле по своей структуре [14], но нерелятивистские квантовый дефект и фазовый сдвиг заменены соответствующими релятивистскими выражениями. При "выключении" потенциала остова фазовый сдвиг $\delta_{k \varkappa}$ обращается в нуль.

Получим выражение для фазы $\Phi_{k \varkappa}(r)$ в квазиклассическом приближении ВКБ для чисто кулоновского потенциала. Рассмотрим сначала случай $l \neq 0$, когда присутствует центробежное отталкивание. Квазиклассическое решение уравнения (6) при $E>1$ имеет вид

$$
\begin{gathered}
f_{k \varkappa}^{\mathrm{WKB}}(r) \sim \sqrt{\frac{E+1}{2 p_{\mathrm{c}}(r)}} \sin \left[\Phi_{k \varkappa}^{\mathrm{WKB}}(r)\right], \quad g_{k \varkappa}^{\mathrm{WKB}}(r) \sim \sqrt{\frac{E-1}{2 p_{\mathrm{c}}(r)}} \cos \left[\Phi_{k \varkappa}^{\mathrm{WKB}}(r)\right], \\
\Phi_{k \varkappa}^{\mathrm{WKB}}(r)=\int_{r_{1}}^{r} p_{\mathrm{c}}\left(r^{\prime}\right) d r^{\prime}+\frac{\pi}{4},
\end{gathered}
$$

где $p_{\mathrm{c}}(r)$ дается выражением (9) с заменой $(10)$, причем $p_{\mathrm{c}}\left(r_{1}\right)=0$. Интеграл в $(12)$ вычисляется с помощью замены переменных $r(\xi)=r_{0}(\epsilon \operatorname{ch} \xi-1), \xi \geqslant 0$, где

$$
r_{0}=\frac{\alpha \mathcal{Z}}{k^{2}}, \quad \epsilon=\sqrt{1+\frac{k^{2} \Lambda_{\varkappa}^{2}}{\alpha^{2} \mathcal{Z}^{2}}} \text {. }
$$

Потребуем, чтобы в асимптотическом значении интеграла (12), при $r \rightarrow \infty$, фаза

$$
\begin{aligned}
\Phi_{k \varkappa}^{\mathrm{WKB}}(r) \approx & k r+\eta \ln (2 k r)+\eta-\eta \ln \sqrt{\eta^{2}+\Lambda_{\varkappa}^{2}}- \\
& -\Lambda_{\varkappa} \arcsin \frac{\eta}{\sqrt{\eta^{2}+\Lambda_{\varkappa}^{2}}}-\frac{\pi}{2}\left(\Lambda_{\varkappa}-\frac{1}{2}\right)
\end{aligned}
$$

совпадала с выражением (11). Используя в (11) формулу Стирлинга для $\eta \gg 1$, что как раз и соответствует квазиклассическому пределу $E-1 \ll 1$, перепишем формулы (11), (13) в виде

$$
\begin{gathered}
\Phi_{k \varkappa}(r) \simeq k r+\eta \ln (2 k r)+\eta-\eta \ln \eta-\pi \omega+\frac{3}{4} \pi, \\
\Phi_{k \varkappa}^{\mathrm{WKB}}(r) \simeq k r+\eta \ln (2 k r)+\eta-\eta \ln \eta-\pi \Lambda_{\varkappa}+\frac{1}{4} \pi .
\end{gathered}
$$

В формуле (11) выбор фаз комплексных чисел проведен в соответствии с условиями

$$
\pi<\arg \left(-l-1-\frac{i \eta}{E}\right)<\frac{3}{2} \pi, \quad-\frac{1}{2} \pi<\arg \left(l-\frac{i \eta}{E}\right)<0 .
$$

Из сопоставления формул (14) и (15) видно, что

$$
\Lambda_{\varkappa}=\omega-\frac{1}{2}=\left\{\begin{array}{lll}
\sqrt{l^{2}-(\alpha Z)^{2}}+1 / 2, & \text { если } & \varkappa=l, \\
\sqrt{(l+1)^{2}-(\alpha Z)^{2}}-1 / 2, & \text { если } & \varkappa=-l-1 .
\end{array}\right.
$$


В нерелятивистском пределе $\alpha Z \rightarrow 0$ мы имеем $\Lambda_{\varkappa} \simeq \mathcal{L}_{l}=l+1 / 2$. Обратим внимание, что параметр $\Lambda_{\varkappa}$ зависит от заряда остаточного иона $Z$, в отличие от нерелятивистского предела.

Случай $l=0(j=1 / 2, \varkappa=-1)$ несколько сложнее, поскольку при $\omega<1$ в квадрированном уравнении Дирака (7) появляется притягивающий потенциал $-\Lambda_{-1}^{2} / 2 r^{2}$. Соответствующий классический импульс вне остова приобретает вид

$$
p(r)=\left[\frac{\Lambda_{-1}^{2}}{r^{2}}+\frac{2 \alpha \mathcal{Z}}{r}-\frac{\alpha^{2} \mathcal{Z}^{2}}{\nu_{n 0}^{2}}\right]^{1 / 2},
$$

что приводит к расходимости интеграла в (12) на нижнем пределе $r_{1}=0$. В этой особой точке, которая совпадает с левой точкой поворота, эффективная потенциальная кривая не линеаризуется, поэтому квазиклассическое решение нельзя представить в виде (12). Для устранения расходимости и вычисления фазового сдвига необходимо найти точное решение вблизи особой точки $r_{1}=0$ в непрерывном спектре и сшить его с квазиклассическим решением вдали от нее в точке $r_{\mathrm{c}}$ (при $k r_{\mathrm{c}} \gg 1$ ).

Асимптотическая фаза (14) при $\varkappa=-1$ формально соответствует решению радиального квадрированного уравнения Дирака (7) с нецелым моментом (8):

$$
\lambda=-\frac{1}{2}+\frac{1}{2} \sqrt{1-4 \Lambda_{-1}^{2}}, \quad 0 \leqslant \Lambda_{-1} \leqslant \frac{1}{2},
$$

в кулоновском потенциале в точке $r_{\mathrm{c}}$. Ограничение на $\Lambda_{-1}$ сверху в (18) диктуется требованием вещественности $\lambda$. Зафиксируем $r_{\mathrm{c}}$ и построим фазу в приближе-

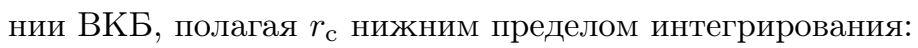

$$
\Phi_{k,-1}^{\mathrm{WKB}}(r)=\Phi_{k,-1}\left(r_{\mathrm{c}}\right)+\int_{r_{\mathrm{c}}}^{r} p_{c}\left(r^{\prime}\right) d r^{\prime},
$$

где $\Phi_{k,-1}\left(r_{\mathrm{c}}\right)$ дается выражением $(14)$, а $p_{\mathrm{c}}(r)$ отличается от $(17)$ заменой $(10)$. При

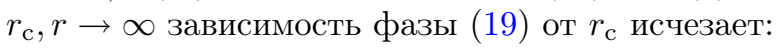

$$
\Phi_{k,-1}^{\mathrm{WKB}}(r)=k r+\eta \ln (2 k r)-\arg \Gamma(\lambda+1+i \eta)-\frac{\pi}{2} \lambda,
$$

что как раз и означает возможность сшивания точного решения с квазиклассическим. При наличии ионного остова к выражению (20) добавляется фазовый сдвиг $\delta_{-1}$, как и в случае $l \neq 0$.

Значение нецелого момента $\lambda$ получается в результате сопоставления квазиклассического решения (20) с асимптотической функцией (14) при условии $\eta \gg 1$ : имеем $\lambda=\sqrt{1-(\alpha Z)^{2}}-1$, откуда

$$
\Lambda_{-1}=\left[\sqrt{1-(\alpha Z)^{2}}-1+(\alpha Z)^{2}\right]^{1 / 2} .
$$

При $\alpha Z \leqslant 1$ все значения $\Lambda_{-1}$ лежат в интервале, заданном в (18). В нерелятивистском пределе $\Lambda_{-1} \simeq \mathcal{L}_{0}=0$. Формулы (16), (21) дают решение поставленной в данном разделе задачи.

\section{3. СПЕКТР РЕЛЯТИВИСТСКОГО ВОДОРОДОПОДОБНОГО АТОМА}

Справедливость замен (16), (21) можно подтвердить, получив квазиклассическим методом энергетические уровни релятивистского электрона в кулоновском потенциале. Как и ранее, рассмотрим отдельно случаи $l \neq 0$ и $l=0$. 
3.1. Случай $l \neq 0$. В соответствии с правилом квантования Бора-Зоммерфельда

$$
\int_{r_{1}}^{r_{2}} p(r) d r=\pi\left(n_{r}+\frac{1}{2}\right), \quad n_{r}=0,1, \ldots,
$$

где классический импульс $p(r)$ определяется формулой $(9)$, являясь непрерывной функцией в обеих точках поворота.

Уравнение (22) необходимо разрешить относительно эффективного главного квантового числа $\nu_{n \varkappa}$. Интеграл в левой части (22) вычисляется при помощи замены

$$
r(\varphi)=r_{0}(1-\epsilon \cos \varphi), \quad 0 \leqslant \varphi \leqslant \pi,
$$

где $\epsilon=\left(1-\varepsilon^{2}\right)^{1 / 2}-$ эксцентриситет классической боровской орбиты,

$$
\varepsilon=\frac{\left|\Lambda_{\varkappa}\right|}{\nu_{n \varkappa}}, \quad \varepsilon<1,
$$

a $r_{0}=\nu_{n \varkappa}^{2} / \alpha \mathcal{Z}$ - ее параметр. В итоге получаем

$$
\nu_{n \varkappa}=\Lambda_{\varkappa}+n_{r}+\frac{1}{2}, \quad n_{r}=0,1, \ldots,
$$

где $\Lambda_{\varkappa}$ определяется соотношением (16). После подстановки (25) в (3) получаем точное релятивистское выражение для энергетического спектра водородоподобного иона [11].

3.2. Случай $l=0$. Случай $l=0(j=1 / 2, \varkappa=-1)$ несколько сложнее, поскольку, как отмечалось выше, $\omega<1$, и в квадрированном уравнении Дирака (7) появляется притягивающий потенциал, пропорциональный $r^{-2}$. Для классического импульса (17) условие Бора-Зоммерфельда (22) неприменимо вследствие логарифмической расходимости интеграла на нижнем пределе $r_{1}=0$. Для получения квазиклассического условия квантования энергий $s$-состояний воспользуемся методом, аналогичным изложенному в работе [15].

Сначала запишем точное решение уравнения (7) для "большой" компоненты, положив $R_{q,-1}(r) \equiv f_{q,-1}(r)$ :

$$
R_{q,-1}(r)=C \exp \left(-\frac{\alpha \mathcal{Z} r}{\nu_{n 0}}\right)\left(\frac{2 \alpha \mathcal{Z} r}{\nu_{n 0}}\right)^{\rho+1 / 2}{ }_{1} F_{1}\left(\frac{1}{2}+\rho-\nu_{n 0}, 1+2 \rho, \frac{2 \alpha \mathcal{Z} r}{\nu_{n 0}}\right)
$$

где

$$
\rho \equiv \sqrt{\frac{1}{4}-\Lambda_{-1}^{2}}, \quad q=\frac{\alpha \mathcal{Z}}{\nu_{n 0}} .
$$

В высоковозбужденных состояниях (при $\nu_{n 0} \gg \alpha \mathcal{Z}$ ), формально соответствующих условию применимости квазиклассического приближения, функция (26) принимает вид [16]

$$
R_{q,-1}(r) \approx C \Gamma(1+2 \rho) \nu_{n 0}^{-\rho-1 / 2} \sqrt{2 \alpha \mathcal{Z} r} J_{2 \rho}(\sqrt{8 \alpha \mathcal{Z} r})
$$

где $J$ - функция Бесселя. В далекой от начала координат области $\sqrt{\alpha \mathcal{Z} r} \gg 1$ для функции Бесселя можно использовать ее асимптотическое разложение [16], и мы получаем

$$
R_{q,-1}(r) \approx \pi^{-1 / 2} C \Gamma(1+2 \rho) \nu_{n 0}^{-\rho-1 / 2}(2 \alpha \mathcal{Z} r)^{1 / 4} \cos \left(\sqrt{8 \alpha \mathcal{Z} r}-\pi \rho-\frac{\pi}{4}\right) .
$$


В нерелятивистской задаче в $s$-состояниях центростремительное притяжение отсутствует $\left(\Lambda_{-1}=0\right)$, и в аргумент косинуса входит фазовый сдвиг $-3 \pi / 4$ [15]. Таким образом, релятивистские эффекты проявляют себя как фазовый сдвиг

$$
\pi\left(\frac{1}{2}-\sqrt{\frac{1}{4}-\Lambda_{-1}^{2}}\right) .
$$

Квазиклассический вид радиальной волновой функции $R_{q,-1}(r)$ удобно строить относительно правой точки поворота $r_{2}$, поскольку ионный потенциал в этой точке непрерывен:

$$
R_{q,-1}^{\mathrm{WKB}}(r)=\frac{A_{q,-1}}{\sqrt{p(r)}} \cos \left(\int_{r}^{r_{2}} p\left(r^{\prime}\right) d r^{\prime}-\frac{\pi}{4}\right),
$$

где $A_{q,-1}-$ некоторая константа. Интеграл в (28) вычисляется с помощью замены

$$
r=r(\varphi)=r_{0}(1+\epsilon \cos \varphi)
$$

где

$$
r_{0}=\frac{\nu_{n 0}^{2}}{\alpha \mathcal{Z}}, \quad \epsilon=\sqrt{\varepsilon^{2}+1}, \quad \varepsilon=\frac{\left|\Lambda_{-1}\right|}{\nu_{n 0}} .
$$

Пределу интегрирования $r_{2}$ соответствует $\varphi=0$. Таким образом,

$$
\int_{r}^{r_{2}} p\left(r^{\prime}\right) d r^{\prime}=\nu_{n 0}\left[\varphi+2 \varepsilon \operatorname{arcth}\left(\sqrt{\frac{\epsilon-1}{\epsilon+1}} \operatorname{tg} \frac{\varphi}{2}\right)-\epsilon \sin \varphi\right] .
$$

Пределу интегрирования $r \rightarrow r_{1}=0$ соответствует $\varphi \rightarrow \arccos (-1 / \epsilon)$. Второе слагаемое в квадратных скобках в (31) ответственно за логарифмическую расходимость интеграла при $r \rightarrow 0$. Действительно, если рассматривать величину $\varphi$ как функцию от $r$ на основании (29), то разложение по малому параметру $r / r_{0}$ дает

$$
\operatorname{arcth}\left(\sqrt{\frac{\epsilon-1}{\epsilon+1}} \operatorname{tg} \frac{\varphi(r)}{2}\right) \approx-\frac{1}{2} \ln \left(\frac{\epsilon r}{2 \varepsilon r_{0}}\right) .
$$

Рассмотрим выражение (31) в области

$$
\frac{\nu_{n 0}^{2}}{\mathcal{Z}} \gg r \gtrsim \frac{\Lambda_{-1}^{2}}{\mathcal{Z}}
$$

Ограничение $r$ сверху обусловлено требованием достаточной удаленности от правой точки поворота, чтобы можно было пренебречь полной энергией электрона (условия применимости квазиклассического приближения требуют $\nu_{n 0} \gg \alpha \mathcal{Z}$ ). Ограничение снизу устраняет логарифмическую расходимость. Для дальнейшего анализа удобно ввести безразмерную переменную $x=\alpha \mathcal{Z} r / \nu_{n 0}^{2}$. Диапазон ее изменения есть

$$
1 \gg x \gtrsim \varepsilon^{2} \text {. }
$$

Ограничение снизу исключает малые значения $\sqrt{x}$.

Разложим выражение (31) по малому параметру $x$ с учетом ограничений $(33)$ :

$$
\begin{gathered}
\varphi=\arccos \frac{x-1}{\varepsilon} \approx \pi-\sqrt{2 x}\left(1-\frac{\epsilon-1}{2 \epsilon x}\right), \\
\sin \varphi=\left[1-\left(\frac{x-1}{\epsilon}\right)^{2}\right]^{1 / 2} \approx \frac{\sqrt{2 x}}{\epsilon}\left(1+\frac{\varepsilon^{2}}{4 x}\right), \quad \operatorname{tg} \frac{\varphi}{2}=\frac{\sin \varphi}{1+\cos \varphi} \approx \sqrt{\frac{2}{x}} .
\end{gathered}
$$


В достаточно широком диапазоне изменения аргумента $\xi \lesssim 1$ можно использовать приближение $\operatorname{arcth} \xi \approx \xi$. В итоге

$$
\int_{r}^{r_{2}} p\left(r^{\prime}\right) d r^{\prime} \approx \nu_{n 0}\left[\pi-\sqrt{2 x}\left(1-\frac{\epsilon-1}{2 \epsilon x}+2(\epsilon-1) \sqrt{\frac{2}{x}}-\sqrt{2 x}\right)\right] .
$$

В этом разложении в соответствии с (33) ведущими слагаемыми будут $\pi \nu_{n 0}$ и слагаемое, пропорциональное $x^{1 / 2}$, поэтому после их удержания мы имеем

$$
\int_{r}^{r_{2}} p\left(r^{\prime}\right) d r^{\prime} \approx \pi \nu_{n 0}-\sqrt{8 \alpha \mathcal{Z} r}
$$

как и в отсутствие центростремительного потенциала.

В результате в интервале (32) радиальная волновая функция в приближении ВКБ принимает вид

$$
R_{q \varkappa}^{\mathrm{WKB}}(r)=2^{-1 / 4} A_{q \varkappa}(\alpha \mathcal{Z})^{-1 / 4} r^{1 / 4} \cos \left(\pi \nu_{n 0}-\sqrt{8 \alpha \mathcal{Z} r}-\frac{\pi}{4}\right) .
$$

Чтобы функции (27) и (34) переходили друг в друга, разность аргументов соответствующих косинусов должна быть кратна $\pi$ :

$$
\nu_{n 0}-\sqrt{\frac{1}{4}-\Lambda_{-1}^{2}}-\frac{1}{2}=n_{r}, \quad n_{r}=0,1, \cdots .
$$

Условие (35) является специфическим случаем условия Бора-Зоммерфельда при наличии особой точки на границе классически разрешенной области. В соответствии с (21) получаем допустимые значения эффективного главного квантового числа:

$$
\nu_{n 0}=\sqrt{1-(\alpha Z)^{2}}+n_{r}, \quad n_{r}=0,1, \cdots .
$$

После подстановки этого выражения в (3) вновь получаем точное релятивистское выражение для энергетического спектра водородоподобного иона [11].

Формулы (25), (36) применимы при $\alpha Z<1$, т. е. при заряде ядра $Z<137$. Таким образом, замены (16), (21) приводят как к точным значениям энергий в состояниях дискретного спектра, так и к точным значениям фаз рассеяния в непрерывном спектре. Последнее обстоятельство не было учтено в работе [9].

\section{4. АСИМПТОТИЧЕСКИЙ ВИД ВОЛНОВОЙ ФУНКЦИИ РЕЛЯТИВИСТСКОГО ЭЛЕКТРОНА В ТЕОРИИ КВАНТОВОГО ДЕФЕКТА}

В нерелятивистском случае радиальная волновая функция валентного электрона в атоме или положительном ионе имеет асимптотический вид

$$
\Psi_{q l m}(\mathbf{r}) \approx C_{q l} q^{3 / 2}(q r)^{\nu_{n l}-1} e^{-q r} Y_{l m}(\mathbf{r} / r), \quad q r \gg 1
$$

В ионах с зарядом высокой кратности (релятивистский случай) радиальная волновая функция валентного электрона имеет вид биспинора (4) с асимптотикой (5).

Безразмерные константы $C_{q l}$ и $C_{q \varkappa}$ определяются зарядом ядра и структурой ионного остова. В работе [17] для нахождения $C_{q l}$ развит метод квантового дефекта, в рамках которого предполагается, что спектр ридберговских состояний в данном ионе известен. В настоящем разделе мы изложим метод нахождения этой константы на основе рассмотренного выше релятивистского квазиклассического приближения. 
4.1. Нерелятивистский квазиклассический метод вычисления $C_{q l}$. Рассмотрим сначала нерелятивистский квазиклассический способ получения константы $C_{q l}$, который формально справедлив при выполнении условия $\nu_{n l} \gg 1$. Пусть электрон с энергией связи (2), совершает финитное движение в кулоновском потенциале с зарядом $Z$. В сферических координатах волновая функция электрона определяется выражением

$$
\Psi_{q l m}(\mathbf{r})=\frac{1}{r} R_{q l}(r) Y_{l m}(\mathbf{r} / r) .
$$

Радиальная волновая функция $R_{q l}(r)$ удовлетворяет радиальному уравнению Шредингера

$$
-\frac{1}{2} \frac{d^{2}}{d r^{2}} R_{q l}(r)+\left[\frac{l(l+1)}{2 r^{2}}-\frac{\alpha Z}{r}\right] R_{q l}(r)=-\frac{\alpha^{2} Z^{2}}{2 \nu_{n l}^{2}} R_{q l}(r) .
$$

В этом уравнении не учитывается влияние на движение электрона короткодействующей части потенциала ионного остова, что как раз и является основным приближением метода квантового дефекта. Короткодействующая часть потенциала влияет только на энергию связанного электрона. Выбор значения этой энергии по данным эксперимента или из более точных расчетов позволяет определить эффективное главное квантовое число $\nu_{n l}$ и тем самым учесть влияние короткодействующего потенциала.

В классически доступной области движения $r_{1}<r<r_{2}$, где $r_{1}$ и $r_{2}-$ классические точки поворота, квазиклассическое решение уравнения (38) определяется выражением

$$
R_{q l}(r)=\frac{A_{q l}}{\sqrt{p(r)}} \cos \left(\int_{r_{1}}^{r} p\left(r^{\prime}\right) d r^{\prime}-\frac{\pi}{4}\right),
$$

где $A_{q l}$ - нормировочный множитель. Пренебрегая короткодействующей частью потенциала, имеем

$$
p(r)=\left[-\frac{\alpha^{2} Z^{2}}{\nu_{n l}^{2}}-\frac{\mathcal{L}_{l}^{2}}{r^{2}}+\frac{2 \alpha Z}{r}\right]^{1 / 2},
$$

а величина $\mathcal{L}_{l}$ задана в $(1)$. Множитель $A_{q l}$ определяется из условия, что в квазиклассическом приближении косинус в (39) быстро изменяется в классически разрешенной области движения, поэтому его квадрат можно заменить средним значением, равным $1 / 2$ :

$$
\left|A_{q l}\right|^{2} \int_{r_{1}}^{r_{2}} \frac{d r}{2 p(r)}=1 .
$$

Для вычисления интеграла (40) проведем замену переменных, аналогичную (23), с той лишь разницей, что вместо $\mathcal{Z}$ используем $Z$, а вместо $\Lambda_{\varkappa}$ используем $\mathcal{L}_{l}$ :

$$
\epsilon=\sqrt{1-\varepsilon^{2}}, \quad \varepsilon=\frac{\mathcal{L}_{l}}{\nu_{n l}}, \quad \varepsilon<1 .
$$

В результате из формулы (40) получаем

$$
A_{q l}=Z \sqrt{\frac{2}{\pi \nu_{n l}^{3}}}
$$


В классически запрещенной области

$$
R_{q l}(r)=\frac{A_{q l}}{2 \sqrt{|p(r)|}} \exp \left(-\int_{r_{2}}^{r}\left|p\left(r^{\prime}\right)\right| d r^{\prime}\right),
$$

и данное выражение должно совпадать с асимптотическим видом волновой функции (37), когда $q r \gg 1$. Интеграл в (43) вычисляется с помощью замены переменных

$$
r(\xi)=r_{0}(\epsilon \operatorname{ch} \xi+1), \quad r_{0}=\frac{\nu_{n l}^{2}}{\alpha Z},
$$

где $\epsilon$ определено в (41). В итоге получается выражение

$$
\begin{aligned}
\int_{r_{2}}^{r}\left|p\left(r^{\prime}\right)\right| d r^{\prime}=q\left\{\varrho(r)+r_{0} \ln \left(r_{0} \epsilon\right)+r_{0} \varepsilon \ln \frac{2 \epsilon}{r_{0}^{2} \varepsilon^{3}}-r_{0} \ln \left[r-r_{0}+\varrho(r)\right]-\right. \\
\left.-r_{0} \varepsilon \ln \frac{2\left[r-r_{0} \varepsilon^{2}-\varepsilon \varrho(r)\right]}{r_{0}^{2} r \varepsilon^{3}}\right\},
\end{aligned}
$$

где $\varrho(r)=\left[\left(r-r_{0}\right)^{2}-r_{0}^{2} \epsilon^{2}\right]^{1 / 2}$. В асимптотически далекой области можно пренебречь $r_{0}$ по сравнению с $r$ всюду, кроме первого слагаемого в правой части (44). Поскольку это слагаемое меняется быстрее всех остальных, а именно линейно растет с ростом $r$, полагаем в этом слагаемом $\varrho(r) \approx r-r_{0}$. Остальные слагаемые изменяются существенно медленнее, логарифмически, и для них достаточно положить $\varrho(r) \approx r$. В итоге выражение $(44)$ упрощается:

$$
\int_{r_{2}}^{r}\left|p\left(r^{\prime}\right)\right| d r^{\prime} \approx q\left(r-r_{0}\right)+\ln \left(\frac{r_{0} \epsilon}{2 r}\right)^{q r_{0}}+\ln \left(\frac{\epsilon}{1-\varepsilon}\right)^{q r_{0} \varepsilon} .
$$

С учетом равенств (42) и (45) выражение (43) в асимптотически удаленной области (при $q r \gg 1$ ) принимает вид

$$
R_{q l}(r) \approx \sqrt{\frac{q}{2 \pi \nu_{n l}}}\left(\frac{\epsilon}{1-\varepsilon}\right)^{q r_{0} \varepsilon}\left(\frac{2 r}{\epsilon r_{0}}\right)^{q r_{0}} e^{q\left(r_{0}-r\right)} .
$$

Сопоставляя формулы (37) и (46), получаем требуемое квазиклассическое выражение для безразмерной константы $C_{q l}$ :

$$
C_{q l}=\frac{e^{\nu_{n l}}\left(2 / \nu_{n l}\right)^{\nu_{n l}}}{\sqrt{2 \pi \nu_{n l}}} L(\varepsilon)
$$

где

$$
L(\varepsilon)=\left(\frac{1-\varepsilon}{1+\varepsilon}\right)^{\mathcal{L}_{l} / 2}\left(1-\varepsilon^{2}\right)^{-\nu_{n l} / 2} .
$$

В $s$-состояниях $L(\varepsilon)=L(0)=1$. Константа $C_{q l}$ получена в квазиклассическом приближении, не требующем, в отличие от метода работы [18], дополнительного условия $l \ll \nu_{n l}$. Это связано с тем, что в работе [18] пренебрегали центробежным отталкиванием. Формула (47) переходит в формулу, приведенную в работе [18], если положить $L(\varepsilon)=1$. Выражение (47) было приведено без вывода в работе [19].

Формула (47) позволяет достаточно просто вычислить коэффициент $C_{q l}$ по известному потенциалу ионизации рассматриваемого иона. Если $\varepsilon>1$, то квазиклассическое приближение оказывается неприменимым, и требуется численное интегрирование уравнений для многочастичных волновых функций, например уравнений Хартри-Фока. 
4.2. Релятивистский квазиклассический метод вычисления константы $C_{q \varkappa}$. Сходство между квадрированным уравнением Дирака (6) и уравнением Шредингера (38) позволяет на основании проведенного анализа утверждать, что при $l \neq 0$, т. е. при наличии центробежного отталкивания, формула для константы $C_{q \varkappa}$ сводится к нерелятивистской формуле (47) с заменой $\mathcal{L}_{l} \rightarrow \Lambda_{\varkappa}$ :

$$
C_{q \varkappa}=\frac{e^{\nu_{n \varkappa}}\left(2 / \nu_{n \varkappa}\right)^{\nu_{n \varkappa}}}{\sqrt{2 \pi \nu_{n \varkappa}}} L(\varepsilon),
$$

где

$$
L(\varepsilon)=\left(\frac{1-\varepsilon}{1+\varepsilon}\right)^{\Lambda_{\varkappa} / 2}\left(1-\varepsilon^{2}\right)^{-\nu_{n \varkappa} / 2},
$$

а параметр $\varepsilon$ определяется выражением (24).

Перейдем к случаю $l=0$. Для определения множителя $A_{q,-1}$ в радиальной волновой функции (28) в классически доступной области получаем, как и ранее, уравнение

$$
\left|A_{q,-1}\right|^{2} \int_{0}^{r_{2}} \frac{d r}{2 p(r)}=1
$$

где $p(r)$ определяется выражением (17). После использования замены (29) находим

$$
A_{q,-1}=\mathcal{Z} \sqrt{\frac{2}{\nu_{n,-1}^{3}}}\left[\Lambda_{-1}+\frac{\pi}{2}\left(1+\frac{2}{\pi} \operatorname{arcctg} \varepsilon\right)\right]^{-1 / 2},
$$

где $\varepsilon$ определено в (30). Отметим, что при $\Lambda_{-1}=0, \varepsilon=0$ выражение (49) переходит в (42).

В классически запрещенной области квазиклассическая радиальная функция связанного состояния, у которой в асимптотически далекой области "большая" и "малая" компоненты биспинора отличаются лишь постоянным множителем,

$$
R_{q,-1}(r)=\frac{A_{q,-1}}{2 \sqrt{|p(r)|}} \exp \left(-\int_{r_{2}}^{r}\left|p\left(r^{\prime}\right)\right| d r^{\prime}\right)
$$

должна совпадать с асимптотической формой решения радиального квадрированного уравнения Дирака:

$$
R_{q,-1}(r) \approx C_{q,-1} q^{3 / 2}(q r)^{\nu_{n,-1}-1} e^{-q r} .
$$

Показатель экспоненты в (50) может быть вычислен по аналогии с нерелятивистским случаем (44) с заменами $Z \rightarrow \mathcal{Z}, \mathcal{L}_{l} \rightarrow \Lambda_{-1}$. Параметры $\epsilon$ и $\varepsilon$ выбираются в соответствии с (30). В асимптотически далекой области $q r \gg 1$

$$
\int_{r_{2}}^{r}\left|p\left(r^{\prime}\right)\right| d r^{\prime} \approx q r-\nu_{n,-1} \ln \left(\frac{2 e q r}{\nu_{n,-1} \sqrt{1+\varepsilon^{2}}}\right)-\nu_{n,-1} \varepsilon \operatorname{arctg} \varepsilon .
$$

Приведем окончательный результат:

$$
C_{q,-1}=\frac{1}{\sqrt{2 \nu_{n,-1}}}\left[\Lambda_{-1}+\frac{\pi}{2}\left(1+\frac{2}{\pi} \operatorname{arcctg} \varepsilon\right)\right]^{-1 / 2}\left(\frac{2 e^{1+\varepsilon \operatorname{arctg} \varepsilon}}{\nu_{n,-1} \sqrt{1+\varepsilon^{2}}}\right)^{\nu_{n,-1}} .
$$



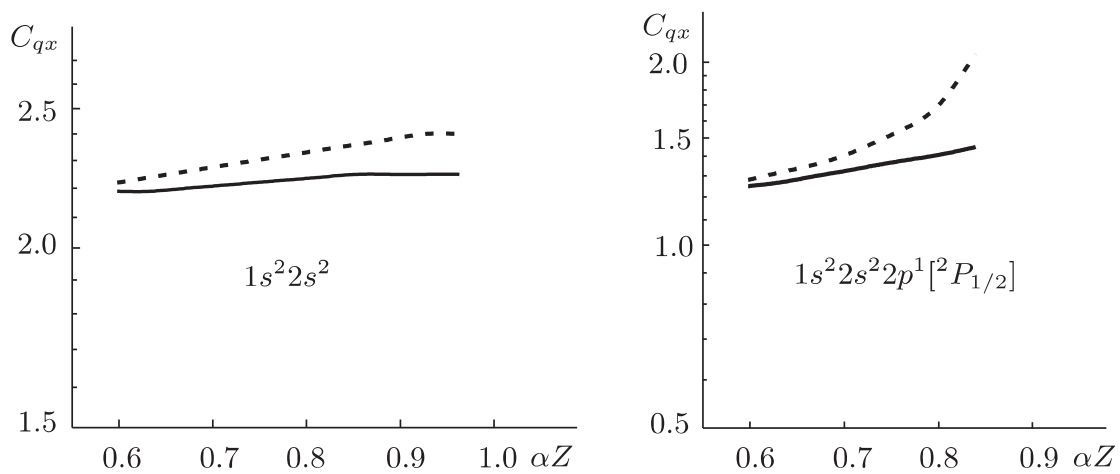

Рис. 1. Значения коэффициента $C_{q \varkappa}$ как функции заряда ядра $Z$ в заданной изоэлектронной последовательности (сплошная линия - релятивистский случай, штриховая линия - нерелятивистский случай).

В нерелятивистском пределе выражение (51) переходит в соотношение (47), в котоpoм $L(\varepsilon)=L(0)=1$.

В качестве примера использования формул (48) и (51) рассмотрим вычисление константы $C_{q \varkappa}$ как функции ионного заряда $Z$ в Ве- и В-изоэлектронных последовательностях с конфигурациями $1 s^{2} 2 s^{2}$ и $1 s^{2} 2 s^{2} 2 p^{1}\left[{ }^{2} P_{1 / 2}\right]$ соответственно. Необходимые значения потенциала ионизации и волнового числа $q$ были получены из решения уравнений Хартри-Фока-Дирака. Результаты расчета представлены на рис. 1. Видно, что релятивистские эффекты становятся весьма существенными для многозарядных ионов. Следует учесть, что вероятность туннельного эффекта во внешнем поле пропорциональна квадрату константы $C_{q \varkappa}$.

Благодарности. Авторы благодарят И.И. Тупицына за любезно предоставленную программу решения уравнений Хартри-Фока-Дирака для многозарядных ионов. Работа выполнена при финансовой поддержке РФФИ (грант № 11-02-00170).

\section{Список литературы}

[1] В. П. Маслов, М. В. Федорюк, Квазиклассическое приближение для уравнений квантовой механики, Наука, М., 1976.

[2] A. S. Kornev, E. B. Tulenko, B. A. Zon, Phys. Rev. A, 68:4 (2003), 043414, 9 pp.; 69:6 (2004), 065401, 2 pp.; 79:6 (2009), 063405, 8 pp.

[3] Б. А. Зон, А. С. Корнев, Е. Б. Туленко, ЖЭТФ, 138:6 (2010), 1043-1059.

[4] N. Milosevic, V.P. Krainov, T. Brabec, Phys. Rev. Lett., 89:19 (2002), 193001, 4 pp.; J. Phys. B, 35:16 (2002), 3515-3529.

[5] В. С. Попов, Б. М. Карнаков, В. Д. Мур, С. Г. Поздняков, ЖКЭТФ, 129:5 (2006), 871-887.

[6] Ch. Jungen (ed.), Molecular Applications of Quantum Defect Theory, IOP, Bristol, 1996.

[7] V. E. Chernov, D. L. Dorofeev, I. Yu. Kretinin, B. A. Zon, Phys. Rev. A, 71:2 (2005), 022505, $11 \mathrm{pp}$.

[8] В. А. Зилитис, Onm. и спектр., 43 (1977), 1017.

[9] В. Ю. Лазур, А. К. Рейтий, В. В. Рубиш, ТМФ, 143:1 (2005), 83-111.

[10] В. А. Давыдкин, Б. А. Зон, Onm. и спектр., 52:4 (1982), 600-606.

[11] Л. Шифф, Квантовая механика, ИЛ, М., 1959. 
[12] З. Флюгге, Задачи по квантовой механике, т. 1, Мир, М., 1974.

[13] В. Б. Берестецкий, Е. М. Лифшиц, Л. П. Питаевский, Теоретическая физика, т. 4: Квантовая электродинамика, Наука, М., 1980.

[14] M. J. Seaton, Proc. Phys. Soc., 88:4 (1966), 801-814.

[15] А.Б. Мигдал, В.П. Крайнов, Приближенные методы квантовой механики, Наука, M., 1966.

[16] М. Абрамовиц, И. Стиган (ред.), Справочник по специалъным функииям с формулами, графиками и математическими таблицами, Наука, М., 1979.

[17] A. S. Kornev, I. Yu. Kretinin, B. A. Zon, Laser Phys., 19:2 (2009), 231-233.

[18] М. В. Аммосов, Н. Б. Делоне, В. П. Крайнов, ЖЭТФ, 91:6 (1986), 2008-2013.

[19] Б. А. Зон, ЖЖЭТФ, 116:2 (1999), 410-417.

Поступила в редакцию 30.05.2011, после доработки 28.07.2011 